\title{
Cluster Based Techniques LEACH and Modified LEACH using Optimized Technique EHO in WSN
}

\author{
Sharad, Sandeep Singh Kang, Deepshikha
}

\begin{abstract}
In the last few years, the Internet of Things (IoT) and the advance wireless networks are becoming very prominent in various domains. Wireless Sensors are facing problems of frequent energy loss which affects to the lifetime of the entire network. There are number of researchers who are working on such energy losses which occur in the wireless sensor nodes by using various approaches. One such method is Low- energy adaptive clustering hierarchy (LEACH) and its number of methods. Despite of various methods of LEACH, there is still immense scope of research as it is highly used in sensor nodes for different scenarios. The emerging growth of energy aware wireless sensor networks for a long time leads to various problems related to the lifetime of nodes in the wireless environment. In our research paper, a new and performance aware approach named Elephant Herd Optimization based Cluster Head Selection is devised and simulated so that the optimization level can be achieved. The nature inspired soft computing approaches are always beneficial for the use of optimization and reduction of various problems which can occur during energy optimization and this is the main focus which is considered in this research work. The main fundamental concept of the cluster head shuffling using EHO and other methods of key exchange are simulated in Contiki-Cooja which is an open source simulator for wireless sensor networks.
\end{abstract}

Index Terms: Advance Wireless Networks, Energy aware Wireless Networks, Elephant Herd Optimization, Energy Optimization.

\section{INTRODUCTION}

Wireless communication (Feeney et al., 2001) integrates the transmission of packets and network signals from the source to destination without any physical infrastructure and hardware environment. This makes the overall communication in integrity and privacy aware mode (Karygiannis et al., 2002). The modes of Wireless Communication include the following aspects in which multiple types of wireless channels are included and thereby the overall communication can be retained for very long time with the higher degree of accuracy and performance.

The classical components in the wireless node comprise of the following segments and the elements:

Revised Manuscript Received on June 22, 2019.

Sharad, Sandeep Singh Kang, Deepshikha : Chandigarh university. Punjab, India.
- Location Finding System

- Mobilizer

- Power Model

- ADC Converter

- Sensor Nodes

- Processor

- Storage Unit

- Transceiver Unit

- Power Supply

- Energy Model

- Security Perspectives

- Security Aspects

- Location Finding Segment

A traditional wireless environment is having the following dimensions and elements

\section{Sender Mote \\ Cluster Head for Data Aggregation \\ Signal Collection Module with Sink Mote}

The source in wireless communication takes the initiatives to transmit the data packet or signal that is further evaluated by the cluster head. The base station is used to have regular communication with cluster head after aggregating the data and signals from the source nodes.

There may be multiple source nodes in the network for communication. The cluster head always keeps track of all the communication in local with its adjacent or associated nodes.

Cluster Head Selection is widely adopted paradigm in the wireless sensor networks whereby the nearby nodes or related nodes are having access to a group head or the cluster head so that they can directly communicate with the local head without energy depletion for the satellite or direct controllers.

Following are the aspects and dimensions as the decision factor for any node to be the cluster head in the network

- Fitness Value

- Appropriate Channel

- $\quad$ Speed 
- Lifetime

- Threshold Aspects

- Energy Value

- Security

- Integrity

- Transparency without Biasing

- Deep Penetration

- Maximum Connections

- Highest Priority

- Cavernous Key Points

- Deep Analysis

- Machine Evaluation

Figure depicts the inherent aspects of the wireless network having the cluster head with the associated cluster node and their communication with the base station so that overall transmission can be done effectively.

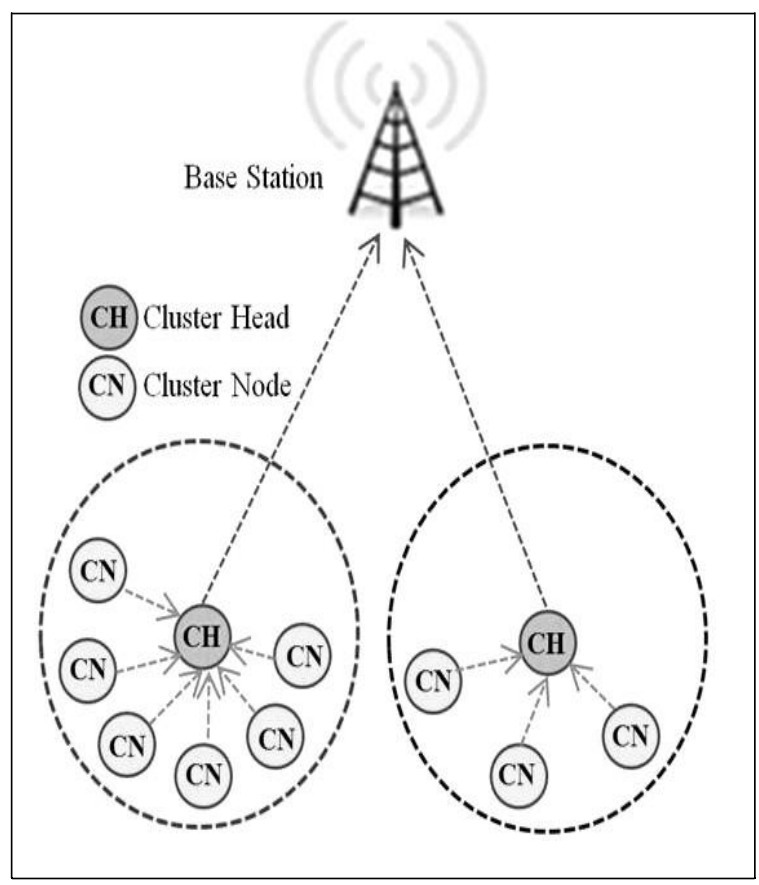

Fig. 1: Base Stations and Clusters

The selection of the cluster head is one of the prominent domains of research since the advent of wireless communication with multiple nodes.

All these nodes communicate with the cluster head so that overall communication can be made free from huge overheads.
Generally, the cluster head is selected from the nearby nodes which are having higher lifetime and energy level. The main focus of our research work is based on the selection of the dynamic cluster head so that the wireless communication can be free from delay or disconnections.

Parameters for the Selection of Cluster Head are:

- Energy Level

- Lifetime

- Security Features

- Maximum Connectivity

- Performance

This research work focuses on the transformation of the electrical energy from an energy source. The decision of the energy transformation is nearly fixed to the decision of the energy source. In the case of energy powered by sun, the change system is the utilization of various sun-oriented boards.

A sun-oriented board acts as a base source and calculate the current produced, its range and power of episode light. Therefore, greater boards with different range or a greater number of sun-based boards are utilized. Our research works are also dealing with energy optimization and this is based on various parameters like battery draining rate and depletion of a node etc.

Advance Wireless Networks using the Internet of Things OpenIoT - OpenIOT refers to the Open Source Platform for the implementation of advance wireless sensor networks with the Internet of Things (IoT) or Cloud of Things (CoT). By this tool, the network environment of different types with multiple nodes of different types can be programmed.

Raspbian - While Raspberry Pi was developed as an educational device, many developers have begun using this credit-card-sized computer for various IOT projects. All the hardware specification is not open source, but most of the software and documentation.

ThinkSpeak - ThinkSpeak is one of the widely used open IoT platforms for collection, storage and visualization of real time sensor data fetched from Arduino, Raspberry Pi, BeagleBone Black and similar boards. By this implementation, live streaming data on cloud can be preserved using channels in ThinkSpeak.

To work with ThinkSpeak, a free account can be created. After successful sign-up on ThinkSpeak, a channel is created on the dashboard and a "Write Key" is generated to be used in code of Arduino. 
The key features of ThinkSpeak includes the following

- Configuration of devices for sending data to cloud-based environment.

- Aggregation of sensor data from different devices and motherboard connected with sensors.

- Visualization of real time sensor data including historical record.

Pre-processing and evaluation of sensor data for predictive analysis and knowledge discovery

\section{[1] Contiki}

http://www.contiki-os.org

IPv6, IPv4, Low Resource, Protothreads, Microcontrollers,

\section{[2] KAA}

https://www.kaaproject.org Data Analytics

Dynamic Updates in Real-Time

The work on energy optimization is quite prominent and widely adopted so that the overall lifetime of the network can be escalated.

Our research work is having key focus on the devising a new soft computing approach for energy optimization.

There are certain points which are covered in our research work

- Identification of the cluster head selection approaches for the energy factors and overall optimization in wireless networks

- Selection of the cluster head with this approach of Elephant Herd Optimization (EHO) as swarm optimization.

- Analysis of the factors in optimization on multiple parameters

The motivation behind this work with EHO is that this approach is a novel approach and not used in the computing domain so far.

In addition, the cluster head selection is also based on the fitness of node that is similar to the elephant selection as head in $\mathrm{EHO}$.

Following are the main points with the use of EHO

- Higher the degree of optimization

- Higher performance in another domain.

- Efficiency in other engineering segments using EHO.

- Similar approach of cluster head selection.

- Node imitation and extraction with fitness.

- Analogous approach of group-based evaluation

- In-built training of dataset with the group values

- Clan based evaluation of dataset

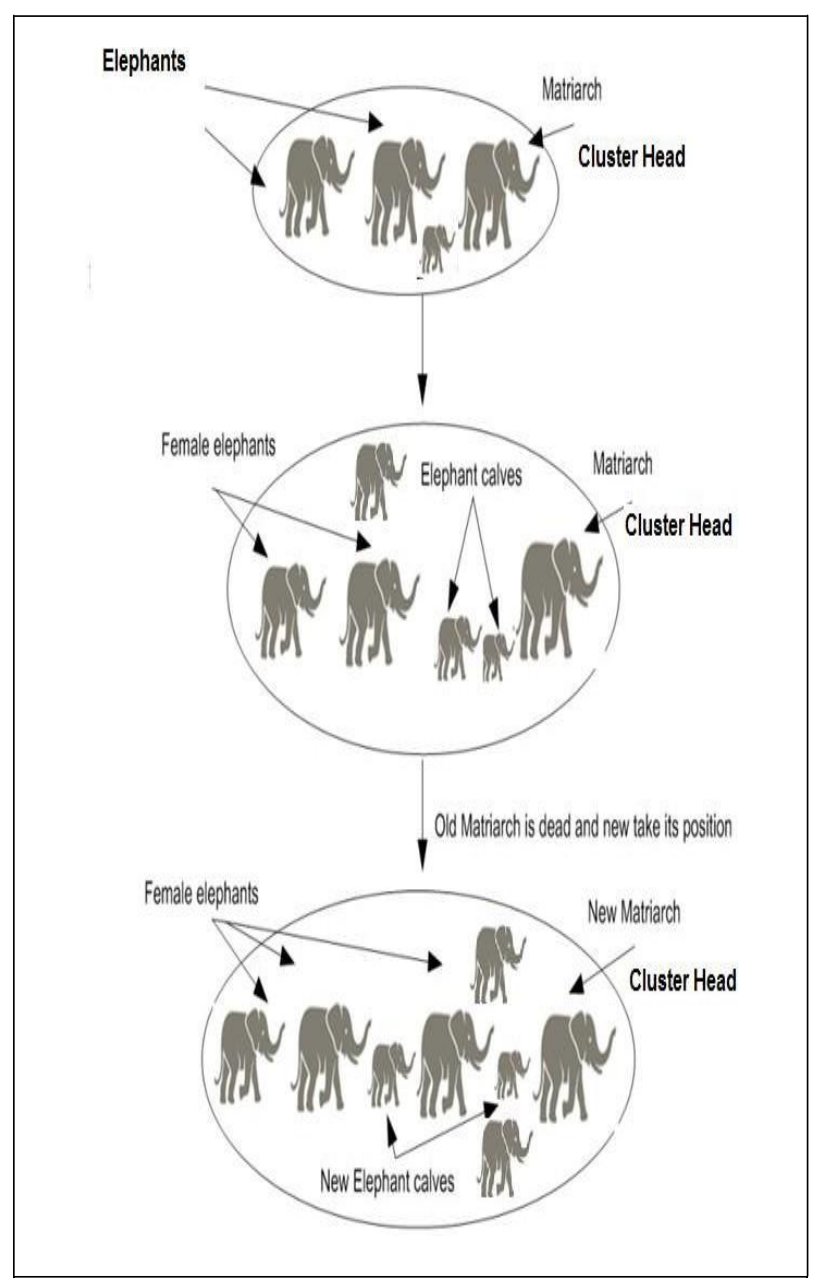

Fig 2: Behaviors of Elephants in the Clan for Selection of Head

\section{REVIEW OF LITERATURE:}

Aydos et al. (1998) presented the work based on the cluster head selection and overall energy optimization. This work is main focus point on the energy and lifetime of the network so that overall communication can be established for long time in the network. The limitations and complexity dimensions of the work includes Prevalent method and cost factor.

Carey et al. (2002) presented the work in view of the nodes, head choice and generally energy enhancement. This work is concentrate on the energy and lifetime of the system with the goal that general correspondence can be set up for long time in the system. The limitations and complexity dimensions of the work includes the higher degree of complexity and resource consumption.

Chan et al. (2003) presented the work in view of the cluster head selection and generally vitality enhancement. This work is key concentrate point on the vitality and lifetime of the system with the goal that general correspondence can be set up to long time in the system. The limitations and complexity dimensions of the work includes cost factor

Gupta et al. (2004) presented a new approach that integrated with energy factor, the lifetime

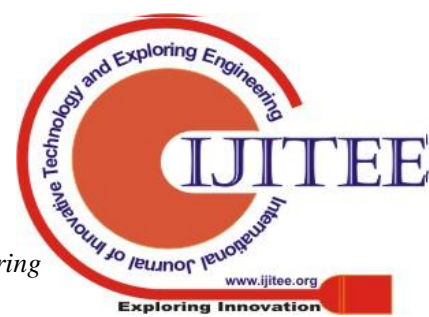


and overall life of the network. This work focused on the identification of key points with the energy consumption and by which way it can be improved. The limitations and complexity dimensions of the work includes Execution time.

Sterbenz et al. (2008) worked on the various limitations and complexity dimensions of the work including running time and complexity. In this approach, the running with influence is picked as an inescapable consequence of contention in the region. Beneficiary based overseeing has been proposed, that makes survey, the energy efficiency, nonappearance of advancement, and multi bounce execution of the check. The beneficiary based on lossless affiliations and gives better results.

The execution-based proposals demonstrate the cross-layer framework at the MAC layer.

The utilization of on off based date-books in a cross- layer controlling and MAC structure is in like the route inspected. In this proposed work, a TDMA-based MAC protocol is devised, where center distributive selects their fitting time openings by considering neighborhood topology information. The arranging tradition furthermore manhandles this information for course establishment.

Adeel (2010) lighted up the three models in wireless sensor networks, which are used as per three distinctive models. In this model system information is gathered into different gatherings. Every social event is made out of one pack head $(\mathrm{CH})$. The diverse $\mathrm{CH}$ gets the different information from the pack part, indicates the identified data and after that sends that particular data to the Base Station. In this, the creator told about the versatile intra bunching directing. In this, the author told that one of the foremost confinements of WSN is noteworthiness. In this examination work this particular issue is recalled and a reaction for bound energy source has been proposed. The proposed course of action is Criticalness Aware Intra Cluster Routing. In this figuring while keeping the degree to the intra bunch correspondence every middle point is not define clearly to other points so that they are organizing the information. A few points are considered in close territory and they perform direct controlling and outside the area focuses to get multihop planning. Hence these closer focuses are not having additional burden on them.

Javed et al. (2013) proposed the wireless Networks that contains the liberal number of fearlessly went on imperativeness obliged sensor center core interests. The work sends the message to incorporate the security with the integrity in energy of wireless environment for the overall communication, secured and improved lifetime of the network.

Roman (2012) presented the use of higher degree of security that is required in the distributed environment. The manuscript focuses on the analysis of threats and attacker models, trust management aspects, authentication, identity with the access control for secured communication. The limitations and complexity dimensions of the work includes execution time and complexity.
Kozlov (2012) underlined the secured Architecture resource optimization and energy consumption dimensions. The limitations and complexity dimensions of the work includes Execution Time and Latency

Jayasudha (2014) underlined that in the wireless sensor network frameworks, sensor nodes continually analyze the sensor network environment and offer data to the base station for further calculations. Since it is a benefit restrictions environment, its framework and lifetime look better with the help of battery support. Therefore, in this paper, a group based restricted desire arrangement is proposed spatial and transitory relationship to have precise data gathering and essential power required by the framework.

Shah (2014) underlined that Wireless Sensor Network (WSN) is growing more in terms of quality and attracted more interest because of is late progress and vary large area. These frameworks are passed on in combination of fields in unattended way and this makes them slanted to different sorts of strikes. Limited resources, like battery life, memory and wireless based channel overhaul inconvenience in execution of security in wireless sensor networks. A couple of pros have suggested their frameworks for realizing security in more efficient way and give diverse symmetric encryption procedures to speed up ready with less memory usage and less stockpiling need.

Zhao (2014) underlined that the principal q-composite key predistribution arrangement (IEEE S\&P 2003) is used basically for secure exchanges as a piece of broad scale wireless sensor frameworks (WSNs).

Ghosal et al. (2015) presented the applications and Implementation of assorted factors in the authentication protocols.

The limitations and complexity dimensions of the work includes effectiveness in the efficient way.

Wang et al. (2015) underlined the work that is EHO based analysis of the particular group or clan that is to be clustered or optimized with the higher degree of accuracy and minimum error.

Li et al., (2016) worked on the cluster head selection with the integration of security with multiple keys for overall communication and higher energy levels. The limitations and complexity dimensions of the work includes integrity.

Moosavi (2016) improved performance speed $97 \%$ with the Integration of new approach of cluster head selection using the presented formulation based on node energy level for getting the better optimization results. The limitations and complexity dimensions of the work includes time-based complexity

Gonzalo (2017) worked on the Internet of Things Approaches and investigated with the deep evaluation on security in IoT. The limitations and complexity 
dimensions of the work includes Susceptibility to Real time attacks.

Meena et al., (2017) introduced a new methodology to solve multi objective distributed energy resource (DER) accommodation problem of distribution systems by combining a technique for order of preference by similarity to ideal solution and an improved elephant herding optimization technique.

\section{Research GAP IN Classical APProach}

- The dynamic cluster head formation based on soft computing and nature inspired approach is required to be implemented for higher degree of optimization and accuracy.

- The shuffling of cluster head with the dying time of current is one of the areas of research.

- The delay in the selection of new cluster head should be minimum and therefore this work is having key focus on this perspective

- LEACH is one of the various approaches that are used for energy efficiency-based routing in Wireless Networks; still this method is under research because of various problems related to energy efficiency.

- In our research work, the integration of nature inspired approach is proposed to enhance the existing results of Improved LEACH.

- There is need to develop and implement a new and effective approach with variant of LEACH is proposed with title EHO based cluster head selection which is called as nature inspired algorithm which is based on LEACH.

- By using this approach, the overall optimization factors can be improved with higher accuracy and performance.

- Need to integrate deep learning.

- Need to analyze the vulnerabilities associated with the energy level.

\section{Problem IDENTIFICATION AND Formulation}

We are performing a detailed evaluation of protocols and methodologies for the energy optimization and lifetime escalation in wireless sensor networks.

We identify and recognize the key points affecting the root cause of higher energy optimization in wireless networks.

We will devise a new approach for energy optimization using nature inspired approach titled Elephant Herd Optimization (EHO) for lifetime escalation. We are trying to perform the implementation and simulation of projected novel approach on recent tool to fetch the real time logs and parameters.
We also perform the comparative analysis of projected EHO based approach with the traditional approach of energy optimization on assorted parameters.

\section{IMPORTANCE OF NEW APPROACH}

- Higher degree of optimization achieved by complete analysis done on multiple nodes so that the cluster head formation can be dynamic

- The changing of the cluster head to avoid any type of attack.

- The nature inspired techniques are always effective in terms of minimum delay and higher performance in the different scenarios

- The repeated flow in the path makes sure that the authentic cluster heads or simply network path for traversing of the data packet via secured path.

- Higher level of energy aware performance and integrity because of the identification and sensing of the malicious traffic and related security factors.

- Secured algorithm to avoid the network intrusion and illegitimate depletion of the energy in the wireless network.

- The proposed method is effective in terms of higher security, integrity and performance.

\section{RESEARCH METHODOLOGY}

1. Evaluation of the approaches and paradigms for the performance and energy levels in the wireless scenarios

2. Analysis of the energy factor during activation of the wireless network

3. Selection of the simulation tool Contiki-Cooja as effective tool for wireless communication and parameters logging

4. Presentation and inclusion of multiple nodes with dynamic topologies and mobility of nodes

5. Integration and implementation of traditional approach for energy optimization

6. Selection, Devising and Implementing Elephant Herd Optimization (EHO) for energy optimization

a. Each sensor node is considered as Elephant.

b. Lifetime and Experience of Elephant is associated with the node energy

7. Selection of the best node in dynamic nature for cluster head

8. Analysis of assorted factors for empirical comparison

- $\quad$ Turnaround Time

- Complexity

- Cost

- Efficiency

- Energy Level 


\section{KEY FACTORS FOR BEST FITNESS}

- Age (Lifetime)

- Energy (Comparison with minimum threshold)

- Prior Experience

- Earlier Attempts

- Clan Updation Operator

- Fitness Score

- Min. Error

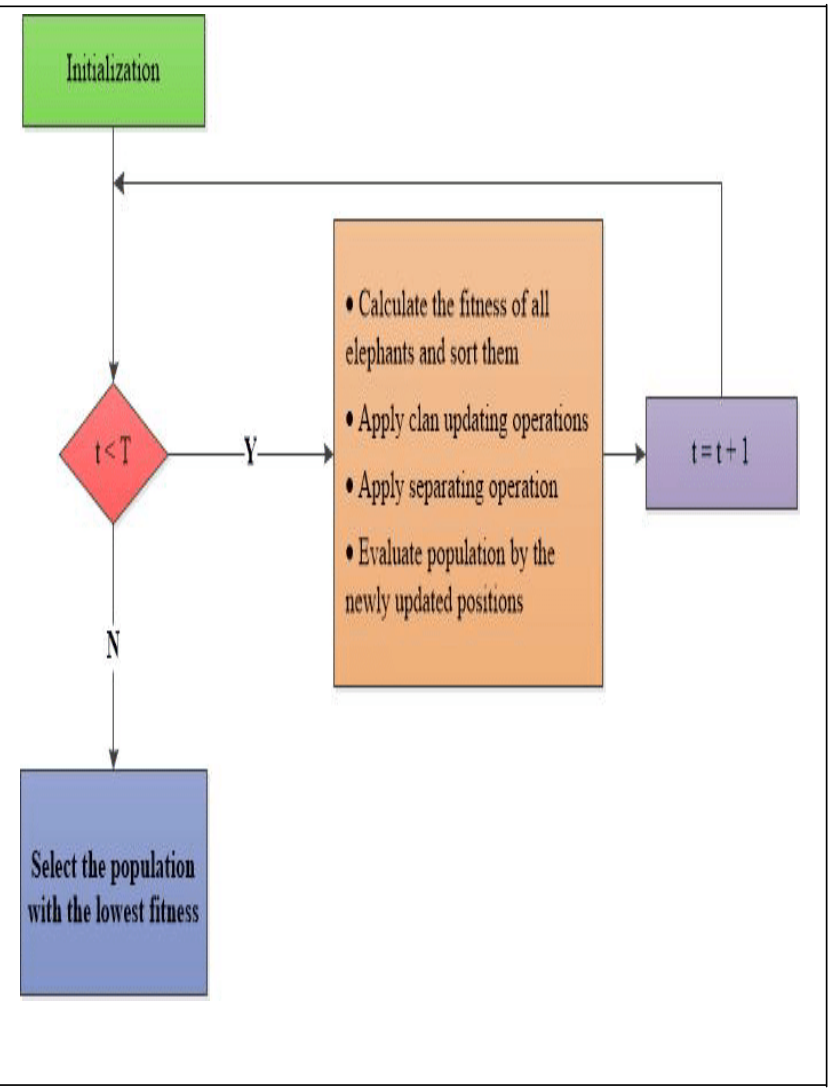

Fig. 3: Elephant Herd Optimization

\section{Simulation Aspects}

1. Nodes Generation using random traffic in ContikiCooja

2. Evaluation of the current energy and radio frequency levels

3. Evaluation of the energy at different phases and lifecycle

4. Implementation and simulation of the mobility with the nodes

5. Integration of EHO for energy optimization

6. Dynamic key exchange for security with the energy level escalation and optimization in the network

\section{Algorithm Elephant Herding Optimization (EHO)}

1. Activation. Inclusion and Spawning of Systems or wireless motes; Changing the Group with Cluster having fitness; Ranking Fitness Score on whole; Setting up the var $\mathrm{g}=1$ and Max. Point of Cluster in Existing Clan (Cluster) MaxCluster
2. WhileBlock i < MaxCluster do

a. Sort OptimalSolutions as per fitness score

3. ForLoopBlock whole Clusters-clans cc do

4. ForLoopBlock whole OptimalSolutions $m$ in the Cluster g perform

a. Revise with Fitness xgi, $m$ and spawn xnew, m by using Fuzzy Fitness

b. Select and Keep better OptimalSolution between xgi, $\mathrm{m}$ and $\mathrm{xnew}, \mathrm{cc}, \mathrm{m}$

c. Revise with Fitness xbest and Inclusion and Spawning of xnew, cc, $m$ using fitness value

d. Select the best OptimalSolution and set in xbest.

\section{END BLOCK FOR LOOP BLOCK}

6. For Loop Block whole ClusterClans $g_{i}$ in the Cluster- set peform

a. Changes of the Less-Fit OptimalSolution in the Cluster-clan cc using Existing score

\section{ENDBLOCK FORLOOPBLOCK}

a. Evaluation of Cluster and calculate the fitness score.

8. ENDBLOCK WHILEBLOCK

9. Return the best fit OptimalSolution $\mathrm{x}_{\text {best }}$ in the ClusterClans

10. If optimal OptimalSolution retrieve. Stop else go to step 3

11. ENDBLOCK

\section{Features OF CONTIKI-COOJA}

Contiki is one of the mostly used operating system under free and open source distribution for simulation of advance wireless networks. To program, control and monitor the wireless environment, the back-end $\mathrm{C}$ programs and related header files can be customized and recompiled to get the desired outcomes. Contiki works on IPv4 and IPv6 networking with the integration of some protocols so that low power chips and radio frequency chips can be connected without any performance issues.

- Free and Open Source Platform

- Back-End C Code

- Low Power Operations with ContikiMAC

- Support for Integration with Hardware

- Sleepy Routers (Battery-Operated Wireless Routers)

- Client-Server Module with claimed World's smallest browser

- Proto Threads (Multi

Threading

Event-Driven

Programming)

Published By: 


\section{Results}

The implementation results are taken from the simulation scenarios developed and simulated in Contiki Platform with Cooja Simulator We compare Existing approach LEACH with EHO on different parameters like energy efficiency, Execution Time, Cost Factor and Complexity and we get improved results in EHO. These comparisons are shown graphically below. By these results we conclude that EHO optimization technique is better than LEACH in terms of these parameters.

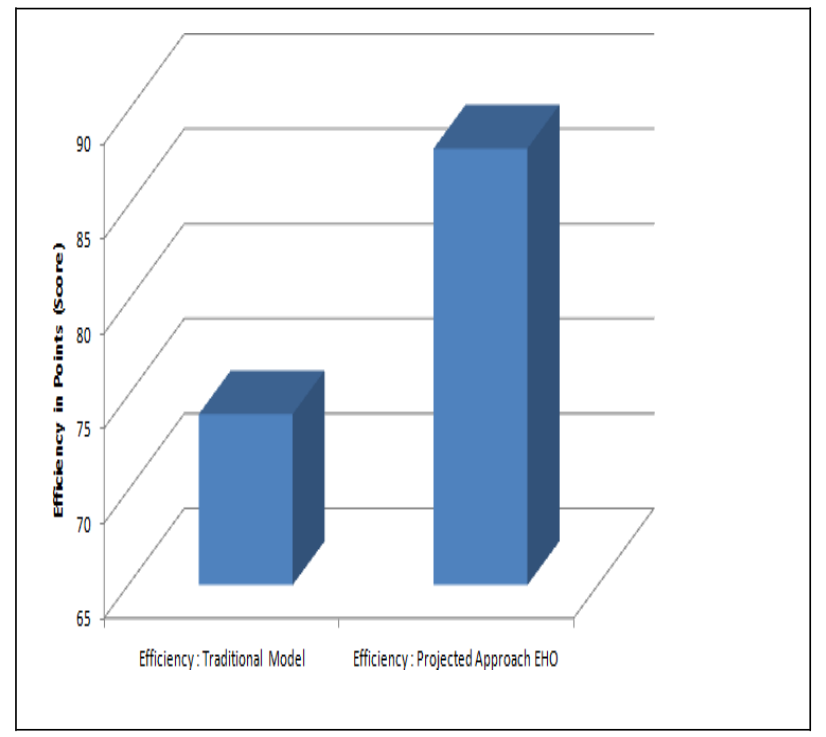

Fig. 4: Comparison of Efficiency Parameter

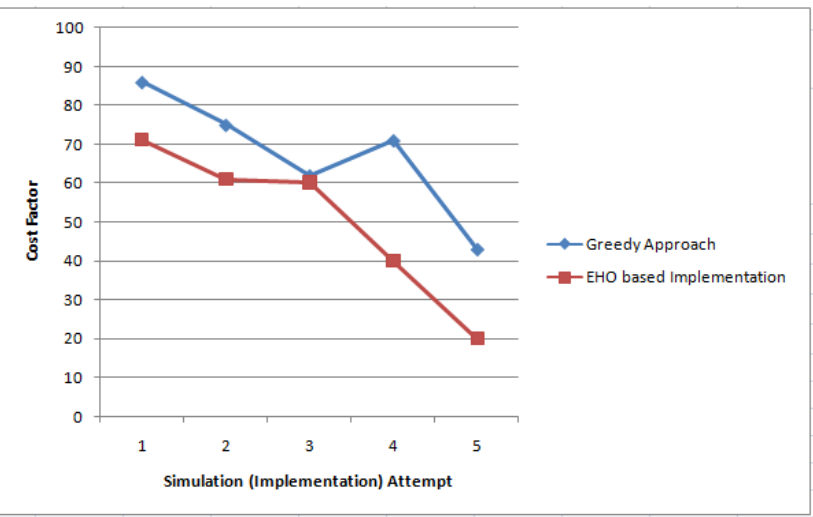

Fig. 5: Comparison of Time Parameter

Table I: Comparative Analysis between Traditional and Projected Approach

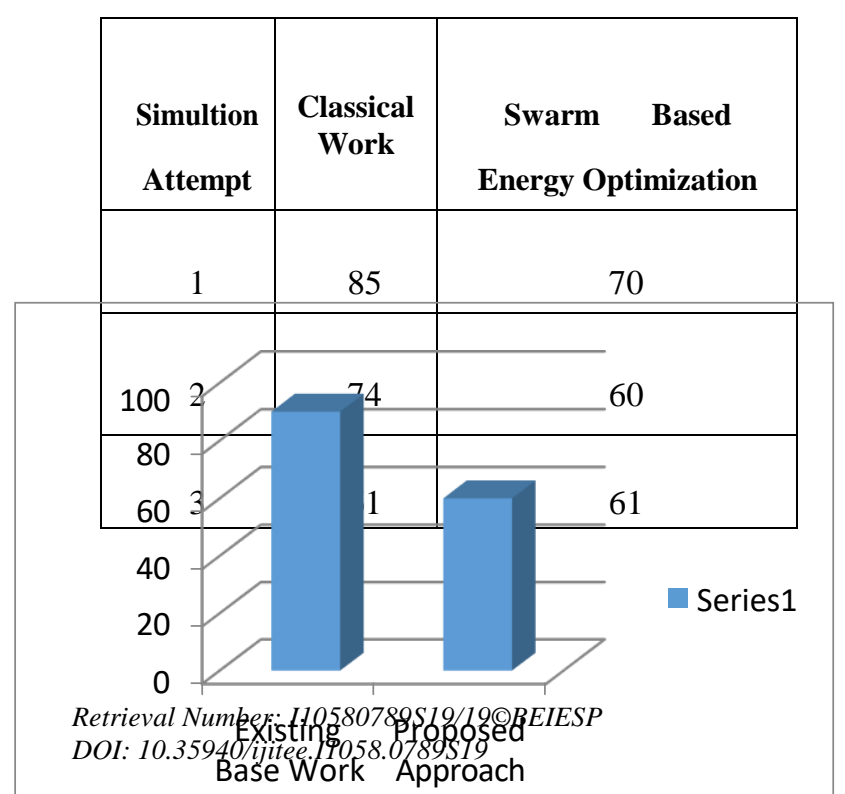

\begin{tabular}{|l|l|l|}
\hline 4 & 72 & 41 \\
\hline 5 & 42 & 24 \\
\hline
\end{tabular}

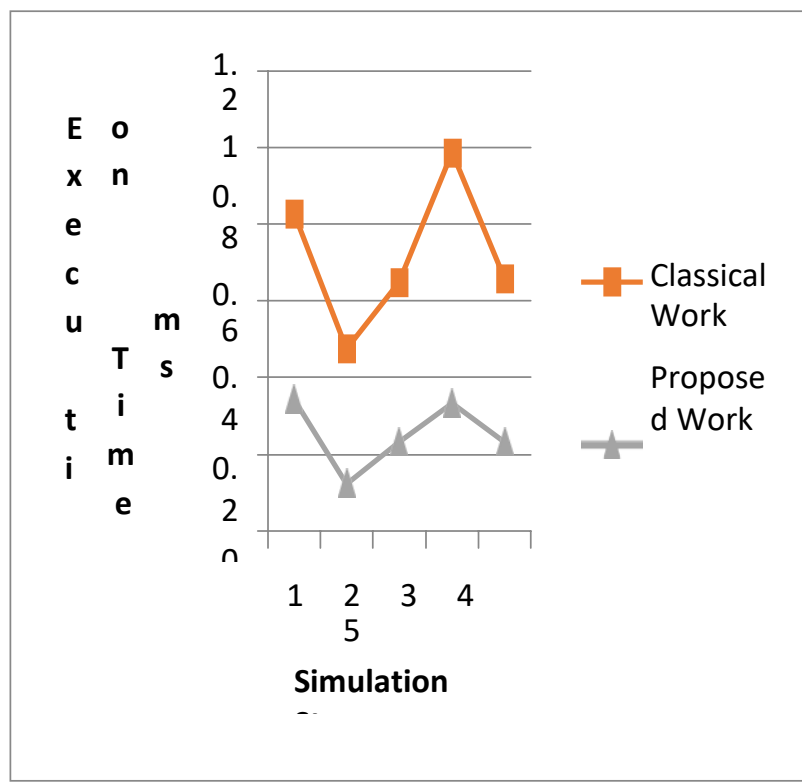

Fig. 6: Comparison of Cost Factor Parameter

Table II: Complexity Level in the Classical and Proposed Approach

\begin{tabular}{|c|c|c|c|}
\hline $\begin{array}{c}\text { Number } \\
\text { of Wireless Nodes }\end{array}$ & $\begin{array}{r}\text { Existing } \\
\text { Approach }\end{array}$ & $\begin{array}{r}\text { Proposed } \\
\text { Approach }\end{array}$ & $\begin{array}{c}\text { Simulation } \\
\text { Iterations }\end{array}$ \\
\hline 50 & 89 & 19 & 10 \\
\hline 50 & 89 & 21 & 30 \\
\hline 20 & 86 & 21 & 50 \\
\hline & & & \\
\hline 20 & 98 & 26 & 70 \\
\hline
\end{tabular}

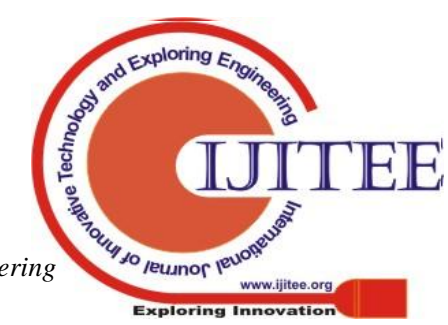


Fig. 7: Comparisons of Complexity and Cost parameters

\section{Flow OF THE RESEARCH Work}

- The different variants based on secured hash approaches gives the clear view of the performance factors in each flavor of EHO.

- EHO is one of the widely used approaches for generation of cluster heads still its performance in distributed and Network based environment gives another dimension to evaluate the performance.

- The key advantage to work on this domain is the assessment of different parameters which are paramount in Ubiquitous Computing.

- Evaluation of variants of EHO using Cooja gives the working scenario on security and integrity in wireless network.

- Network based implementation of EHO on Cooja provides the performance in distributed environment.

- Complexity Level in EHO is less as compared to Existing Approach.

- Complexity Level in the Classical and Proposed Approach

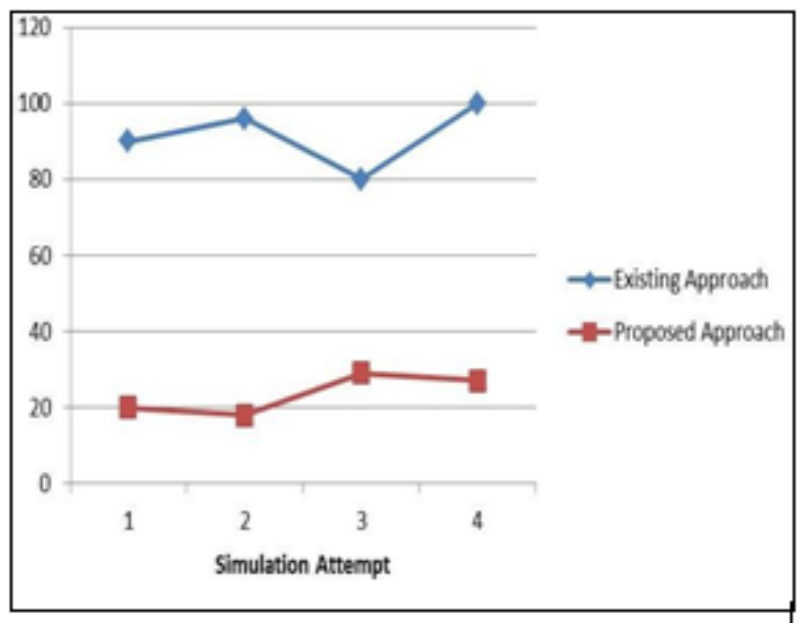

Fig. 8: Comparison of Complexity Parameter
Fig. 9: Comparison of Complexity Parameter

- From the results and outcome, it is clear that the projected approach of EHO is giving better performance as compared to the previous approach.

- The research on wireless based secured transportation systems is still in progress and being integrated by assorted automobile companies. Still, there is colossal span for the development of novel, unique and effective algorithms.

\section{Conclusion}

Our research concludes with the presentation and validation of the fact from simulation that is based on soft computing approach. It is quite effective in multiple parameters even in the ad hoc environment scenarios. The implementation done in Cooja platform gives the valuable results and the approach is superior as compared to the other variants of traditional approaches. The Proposed work helps to overcome the congestion problem arises during packet transmission in wireless sensor network, our technique provides the solution to the mobile sensor nodes under various scenarios and the obtained results were improved comparing to the proposed techniques.

The determination of group head is one of the unmistakable spaces of research since the appearance of wireless correspondence with the numerous nodes. Every one of these nodes communicates with the bunch head so in general correspondence can be made free from colossal overheads.

For the most part, the bunch head is chosen from the close-by nodes which are having higher lifetime and energy level. This research work is having the main concentrate point on the choice of the dynamic group head with the goal that the wireless correspondence can be free from postponement or detachments.

There are main focuses which are surrounding in this examination work: Identification of the bunch head determination approaches for the energy elements and generally speaking improvement in the wireless systems

- $\quad$ Selection of the bunch head with new approach of Elephant Herd Optimization (EHO) as swarm enhancement

- Analysis of the components in improvement on numerous parameters

- $\quad$ Node energy and

Published By: 
elevation

- $\quad$ Predictive evaluation of energy

- Elephant Herd Optimization based energy optimization

- Swarm based Optimization

The inspiration driving this work with EHO is that this approach is a novel approach and not utilized as a part of the processing space to colossal degree up until this point. Moreover, the bunch head determination is likewise in view of the wellness of node that is like the elephant choice as head in EHO.

\section{FUTURE SCOPE}

The work presents the use of Elephant Herd Optimization (EHO) as the optimization of energy levels. This approach is used for the escalation of energy and lifetime in the wireless network and similar approaches can be used for further evaluations and optimization.

There are assorted hyper-heuristic and swarm based techniques which can be further integrated in hybridization as follows

- Flower Pollination Approach

- River Formation Dynamics

- Bat Approach

- Bull Optimization

- Optics Paradigm

- Golden Ball Approach

- Eagle Strategy

- Altruism Approach

- Black Holes Techniques

- Mine Blast Optimization

- Krill Herd Optimization

- Group Search Optimization (GSO)

- Cuckoo Search (CS)

- Cultural Approaches

- Strawberry Approach

- Darwin based Optimization

- Simulated Annealing

- Chemical Fermentation and many others
$6,418,327,2002$

4. Chan, and Perrig, "Security and privacy in sensor network computer", 36(10), pp.103-105, 2003

5. Gupta, Stebila, Fung, Shantz, Gura, and Eberle, , Augus "Speeding up Secure Web Transactions Using Elliptic Curve Cryptograph In NDSS", 2004

6. Sterbenz, Medhi, Monaco, Ramamurthy, Scoglio, Choi,B. Evans, J. Gruenbacher, Hui, Kaplow, and Minden,.GpENI: "Great Plains Environment for Network Innovation" Proposal).2008 ITTC technical report ITTC-FY2009TR-0061349-01, The University of Kansas.

7. Roman, Zhou, and Lopez, "On the features and challenge of security and privacy in distributed internet of things," Computer Networks, vol. 57, n 10, pp 2266-2279, 2013.

8. Jayashree, Arumugam, Anusha, and Hariny, A. (2006),“Apri On the accuracy of centroid based multilateration procedure for location discovery in wireless sensor network In Wireless and Optical Communications Networks, IFIP International Conference on (p 6-pp). IEEE. 2006

9. Uckelmann Harrison Michahelles "An architectural approach towards the future internet of things", Springer Berlin Heidelberg, pp 1-24, 2011.

10. Zhang, Liang, Lu, \& Shen, "Sybil attacks and their defenses in the internet of things." IEEE Internet of Things Journal, (5), pp 372-383, 20141.

11. Wang, Deb Coelho,"Elephant herding optimization In Computational and Business Intelligence (ISCBI)", 3rd International Symposium on Dec 7 (pp 1-5). IEEE, 2015.

12. Trappe, Howard, and Moore, "Low-energy security: Limits and opportunities in the internet of things", IEEE Security and Privacy, vol. 13, pp 14-21, 2015.

13. Said, Masu "Towards internet of things: Survey and future vision". International Journal of Computer Network pp.1-7, 2015.

14. Rehiman and Veni, "A secure authentication infrastructure for IOT enabled smart mobile devices - an initial prototype," Indian Sc Techno vol. 9, pp 1-6 2016 .

15. Li, Zheng, and Jin, "Secure and efficient data transmission in the Internet of Things," Telecommunication Systems, vol. 62, n 1, pp 111-122, 2016.

16. Mollah, Azad, and Vasilakos, "Secure data sharing and searching at the edge of cloud-assisted internet of things," IEEE Cloud Computing, vol. 4, pp 34-42, 2017.

\section{AUTHORS PROFILE}

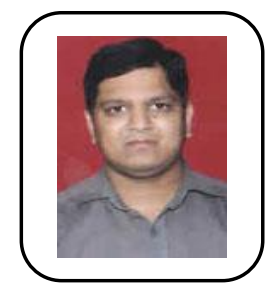

Dr.Sharad has been working as Assistant Professor in CSE Department at Chandigarh University,Gharuan (Mohali), Punjab (India).He has done his B.Tech, M.E and Ph.D in CSE. He has more than 15 years of teaching experience in various Engineering colleges. He has published 35 Research papers in International/National Journals and Conferences. He has published 8 books on different subjects of CSE.

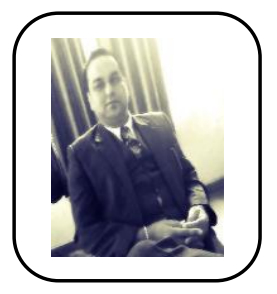

Dr. Sandeep Singh Kang has been working as Professor in Computer science \& Engineering at Chandigarh University Gharuan (Mohali), Punjab (India). He did his B.Tech, M.Tech and Ph.D in Computer Science and Engineering. He has 16 years of teaching experience in the field of engineering education. $\mathrm{He}$ has published 70 Research papersin International/National Journal and Conferences. He has published one book on Network Security. He is the life member of ISTE.

\section{REFERENCES}

1. Feeney, L. and Nilsson, M. "Investigating the energy consumption of a wireless network interface in an ad hoc networking environment". IEEE INFOCOM 3:1548- 1557 vol.3, 2001

2. Aydos, Sunar, and Koc, C "An elliptic curve cryptographybased authentication and key agreement protocol for wireless communication" in 2nd International Workshop on Discrete Algorithms and Methods for Mobile Computing and Communications Symposium on Information Theory, 1998

3. Carey, Peragine, Dziadek, Herscovici, and Ryan, Spike Broad andSystemic,"Methodsand determining an ptimum sector distribution within a coverage area of a wireless communication system" U. Patent

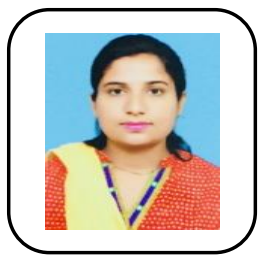

Er Deepshikha has been working as an Assistant Professor in Chandigarh University from July 2016.She has done B tech from MITS Lakshmangarh Sikar and M.Tech from Jamia Hamdard Delhi. She has published two chapters in IGI journals and has published various research papers in International/National Journals.

Published By:

Blue Eyes Intelligence Engineering

\& Sciences Publication 
